\title{
Identification of a CCG-enriched expanded allele in DM1 patients using
}

4 Yu-Chi Tsai ${ }^{1}$, Laure de Pontual ${ }^{2}$, Cheryl Heiner ${ }^{1}$, Tanya Stojkovic ${ }^{3}$, Denis Furling ${ }^{2}$, Guillaume

5 Bassez ${ }^{2,3}$, Geneviève Gourdon² and Stéphanie Tomé2

$7 \quad$ 1Pacific Biosciences, Menlo Park, CA 94025, USA

8 2Sorbonne Université, Inserm, Institut de Myologie, Centre de Recherche en Myologie, F-

975013 Paris, France

$10{ }^{3}$ Centre de Référence des Maladies Neuromusculaires Nord-Est/Île-de-France, Institut de

11 Myologie, GHU Pitié-Salpêtrière, AP-HP, Paris, France.

12

13 Corresponding authors: stephanie.tome@inserm.fr

14 Repeat Expansions \& DM

15 Centre de Recherche en Myologie, UMRS974

16 Sorbonne Université / INSERM / AIM

17 47, bld de l'hôpital - G.H. Pitié-Salpétrière - Bâtiment Babinski

1875651 Paris cedex 13, France

19 Tél : 033142165716 / Fax : 033142165700 


\section{Abstract}

Myotonic dystrophy type 1 (DM1) exhibits highly heterogeneous clinical manifestations caused by an unstable CTG repeat expansion reaching up to 4,000 CTG. The clinical variability depends on CTG repeat number, CNG repeat interruptions and somatic mosaicism. Currently, none of these factors are simultaneously and accurately determined due to the limitations of gold standard methods used in clinical and research laboratories. An amplicon method for targeting DM1 locus using Single-Molecule Real-Time sequencing was recently developed to accurately analyze expanded alleles. However, amplicon-based sequencing still depends on PCR and the inherent bias towards preferential amplification of smaller repeats can be problematic in DM1. Thus, an amplification-free long-read sequencing method was developed using the CRISPR/Cas9 technology in DM1. This method was used to sequence the DM1 locus in patients with CTG repeat expansion ranging from 130 to $>1000$ CTG. We showed that elimination of PCR amplification improves the accuracy of measurement of inherited repeat number and somatic repeat variations, two important key factors in the DM1 severity and age at onset. For the first time, an expansion composed of over $85 \%$ CCG repeats was identified using this innovative method in a DM1 family with an atypical clinical profile. No-Amplification targeted sequencing represents a promising method that can overcome research and diagnosis shortcomings, with translational implications for clinical and genetic counseling in DM1. 


\section{Introduction}

Short tandem repeats are an important source of genetic variation and phenotypic variability in disease and health, that are not always well characterized and understood due to their complexities. Among these repeated elements, unstable repeat expansions are associated with more than twenty diseases including the complex and variable myotonic dystrophy type 1 (DM1) disorder ${ }^{1}$. DM1 is caused by an unstable CTG repeat expansion in the $3^{\prime}$ UTR of the dystrophia myotonica protein kinase (DMPK) gene, which usually increases across generations and over time in somatic tissues ${ }^{2,3}$. In DM1, longer expanded alleles are usually associated with a worsening of clinical severity and an earlier age of onset ${ }^{4}$. This anticipation phenomenon is particularly obvious in $\mathrm{DM} 1^{5}$. DM1 is mainly characterized by an unusually broad clinical spectrum of symptoms divided into five distinct clinical forms ranging from late onset to the congenital forms which are often associated with the largest size of inherited disease-associated allele ${ }^{4}$. Facial dysmorphisms, muscle weakness and cognitive impairment are more frequent symptoms at an earlier onset, while cardiac defects and cataracts are more common in DM1 patients with later forms of the disease ${ }^{4}$.

In DM1, it is laborious to diagnose patients and classify them into distinct clinical categories based exclusively on mutation status and size of CTG repeats when known, for several reasons. First, the long-expanded allele and the precise number of CTG repeats are complicated to identify and measure by conventional methods, particularly for the larger repeat expansions ${ }^{6,7}$. Second, DM1 patients exhibit high clinical and genetic variability which cannot be exclusively explained by the size of the CTG repeats ${ }^{4}$. Third, additional factors such as somatic mosaicism are important disease modifiers contributing to the high genotype and phenotype variability observed in DM1. Somatic mosaicism biased towards expansions contributes to the progressive nature of the various DM1 symptoms and also to the variation 
in the age of onset ${ }^{8-11}$. Forth, the majority of DM1 patients inherit pure CTG repeat expansion.

However, more than $8 \%$ of known DM1 patients carry interruptions that vary in type (CCG,

CAG, CTC and CGG) and number between families and also among individuals of the same family ${ }^{12}$. Interruptions are frequently associated with intergenerational contractions and stabilization of the CTG repeat as well as milder DM1 symptoms and / or additional symptoms ${ }^{11,13-22}$. Fifth, single-nucleotide polymorphisms in the DNA mismatch repair gene MSH3, required for maintenance of genomic integrity, have been shown to reduce levels of somatic mosaicism and are associated with delayed onset in DM1 patients ${ }^{23,24}$. The contribution of CTG repeat length, somatic mosaicism, structural variants and/or modifier genes on the DM1 genotype and phenotype remains poorly understood due to technical difficulties in analyzing them.

81 Currently, the molecular diagnostic procedure used in DM1 genetic testing is divided into two steps $^{6,7}$. The first is to detect or rule out possible DM1 expansions using PCR and fragmentlength analysis (Table 1). This method cannot differentiate between individuals homozygous for a normal allele and individuals whose CTG repeat expansion could not be amplified by PCR. A second step is therefore necessary to identify large expanded alleles and estimate the approximate size using Southern blotting. All of these methods are time-consuming procedures that yield no information on somatic mosaicism and structural variants of expansion, two important prognostic parameters. Furthermore, triplet-repeat primed PCR (TP-PCR) is also used to directly detect the presence of an expanded allele ${ }^{25}$. However, no size repeat can be estimated by this method, which leads to a loss of information necessary for 91 carrying out genetic counseling. To date, TP-PCR is the only diagnostic method which makes it possible to reveal variant repeats in the first $100 \mathrm{bp}$ within the $5^{\prime}$ and $3^{\prime}$ end of CTG array. 
expansions of more than 200-300 CTG repeats and to give the exact number and type of interruptions, regardless of the size of the repeats. Diagnostic laboratories generally use several methodological approaches to detect CTG repeat expansion and roughly estimate the size of the repeat which leads to long diagnostic delays that can exceed 3 months. Unfortunately, no diagnostic method can estimate somatic mosaicism which is an important parameter to predict disease progression ${ }^{8-11}$. Today, the most accurate method to assess the inherited CTG repeat expansion size and the level of somatic mosaicism is the Small-Pool PCR

101 (SP-PCR) ${ }^{9,26}$. SP-PCR, PCR on successive DNA dilutions, is a time-consuming and fastidious

102 method, exclusively used in the research laboratories. The PCR amplification bias towards

103 shorter repeats and the difficulties inherent to the amplification of the GC rich DM1 locus by

104 this method remain a concern, which needs to be addressed by a new widely available 105 method. In addition, neither the SP-PCR nor Southern blotting can detect CNG interruptions 106 within the repeats, which can lead to inaccurate genetic analysis and genotype-phenotype 107 associations. Currently, molecular diagnostic tests used in diagnostic laboratories to analyze 108 the DM1 locus may induce loss of information and bias in the interpretation of genetic data, 109 especially when patients carry large CTG repeats. It is therefore essential to accurately 110 measure the number of CTG repeats, structural variants and somatic mosaicism in DM1 111 patients using a fast and accurate method to provide better diagnosis and prognosis with 112 minimal delay. Pacific Biosciences' (PacBio) targeted single-molecule long-read real-time 113 sequencing from PCR products was recently described to analyze the DM1 locus in 114 patients ${ }^{16,27}$. This method allows accurate counting of repeat numbers in large expanded 115 alleles, and can simultaneously evaluate the degree of somatic mosaicism and identify 116 interruptions ${ }^{27}$. However, amplicon-based sequencing relies on PCR and is still subjected to 117 the inherent preferential amplification of smaller repeats, which introduces a confounding 
118 and undesirable bias in repeat sizing. To overcome this limitation, we developed a robust

119 amplification free-targeted long-read sequencing for DM1. Using this method, measurement

120 of CTG repeat length and somatic mosaicism is improved in DM1 patient samples. The second

121 advantage of this method is that CNG interruptions are easily detected regardless of the size

122 of the CTG repeat expansions. Strikingly and for the first time, we identified triplet repeat expanded alleles composed of more than $85 \%$ of CCG in DM1 patients for whom amplification

124 of the triplet repeat expansion failed by PCR and TP-PCR.

125

\section{Materials and Methods}

129 DM1 participants were recruited by the Genetics Department of Nantes Hospital, the Genetics

130 Department of the Necker-Enfants Malades Hospital, the DM-Scope registry and the

131 Neuromuscular Disease Reference Center of Pitié-Salpêtrière Hospital in France. Written

132 informed consent was obtained from all participants. Genomic DNA samples were initially

133 genotyped for DM1 using conventional PCR, TP-PCR, or Southern Blot ${ }^{6,7}$. DNA was extracted

134 in diagnostic laboratories. High molecular weight (HMW) DNA was extracted from

135 immortalized lymphoblastoid cells by the Monarch ${ }^{\circledR}$ Genomic DNA Purification Kit from New

136 England Biolabs.

138 Design of Guide RNAs for cas9 digestion

139 Human Hg19 reference sequences surrounding the DM1 repeat region were used to design

140 Cas9 CRISPR RNA (crRNA) sequences. Candidate target sequences were generated using the 141 GPP sgRNA Designer webtool on the Broad Institute website 
142 (https://portals.broadinstitute.org/gpp/public/analysis-tools/sgrna-design). Two upstream

143 and three downstream top ranking candidate sequences which could generate approximately

$1442 \mathrm{~kb}$ target fragments were custom synthesized and tested in targeted sequencing

145 experiments with HEK293 genomic DNA. The combination of upstream and downstream

146 crRNAs that produced the highest sequencing yield was selected for this study. The final crRNA

147 sequences are the DM1-L1-crRNA sequence: 5'- CCCCATCGGGACAACGCAGA-3' and the DM1-

148 R1-crRNA sequence: 5'- GGGCGTGTATAGACACCTGG-3' which generates a target capture

149 region of $2361 \mathrm{bp}$.

151 SMRT bell library preparation

152 Preparation of the SMRTbell ${ }^{\circledR}$ library was performed according to the Procedure and Checklist-

153 No-Amp Targeted Sequencing utilizing the CRISPR-Cas9 system, available on the PacBio

154 website (https://www.pacb.com/wp-content/uploads/Procedure-Checklist-No-Amp-

155 Targeted-Sequencing-Utilizing-the-CRISPR-Cas9-System.pdf). Approximately 3-5 $\mu$ g of native

156 genomic DNA were dephosphorylated with $0.05 \mathrm{U} / \mu \mathrm{l}$ of shrimp alkaline phosphatase (rSAP)

157 (New England BioLabs) to reduce the amount of off-target molecules in the final SMRTbell ${ }^{\circledR}$

158 library. Guide RNAs (gRNAs) were formed by annealing crRNAs (Integrated DNA Technologies)

159 to tracrRNAs (Integrated DNA Technologies) at a 1:1 ratio and $5 \mu \mathrm{M}$ concentration. The

160 sequences of each crRNA targeting DM1 and various control loci used in the experiments are

161 shown in Table 2. The gRNA-Cas9 complex was prepared by incubating 400nM gRNA with

$162400 n M$ Cas9 nuclease (New England Biolabs) for $10 \mathrm{~min}$ at $37^{\circ} \mathrm{C}$. Dephosphorylated genomic

163 DNAs were digested with the gRNA-Cas9 complex for 1 hour at $37^{\circ} \mathrm{C}$. DM1 and control gRNA

164 were multiplexed in the same digestion reaction. Cas9 digestion products were purified using

165 a 0.45X volume of AMPure PB beads (Pacific Biosciences). Barcoded adapters recommended 
by PacBio were ligated to purified Cas 9 digestion products using $0.4 \mu \mathrm{M}$ barcode adapters and 0.9U/ $\mu$ I T4 DNA ligase (Thermo Fisher Scientific) to form a library of symmetric SMRTbell template molecules. The SMRTbell ${ }^{\circledR}$ library was purified using a $0.45 \mathrm{X}$ volume of AMPure ${ }^{\circledR} \mathrm{PB}$ beads (Pacific Biosciences, Menlo Park, CA, USA). Failed ligation products and gDNA fragments were removed by nuclease treatment using 1.2U/ul of Exonuclease III (New England BioLabs) and an Enzyme Clean Up Kit (Pacific Biosciences, Menlo Park, CA, USA) for 2 hours at $37^{\circ} \mathrm{C}$.

172 After nuclease treatment, the SMRTbell ${ }^{\circledR}$ library was incubated with $41 \mu \mathrm{g} / \mathrm{mL}$ SOLu-Trypsin

173 (Sigma-Aldrich) for 20 minutes at $37^{\circ} \mathrm{C}$. The nuclease-treated SMRTbell ${ }^{\circledR}$ library was purified

174 using a $0.45 \mathrm{X}$ volume of $\mathrm{AMPure}{ }^{\circledR} \mathrm{PB}$ beads for the first purification and a $0.42 \mathrm{X}$ volume of 175 AMPure ${ }^{\circledR}$ PB beads for the second purification. Finally, the purified SMRTbell library was 176 resuspended in $6.3 \mu \mathrm{l}$ of elution buffer for targeted $\mathrm{SMRT}^{\circledR}$ sequencing.

$178 \quad$ Targeted SMRT sequencing

179 Following the Procedure and Checklist-No-Amp Targeted Sequencing Utilizing the CRISPR-Cas9

180 System (https://www.pacb.com/wp-content/uploads/Procedure-Checklist-No-Amp-

181 Targeted-Sequencing-Utilizing-the-CRISPR-Cas9-System.pdf), final SMRTbell ${ }^{\circledR}$ libraries were

182 annealed with Sequencing Primer v4 from the No-Amp Accessory Kit (Pacific Biosciences,

183 Menlo Park, CA, USA) and bound with Sequel ${ }^{\circledR}$ DNASequencing Polymerase 3.0 in the Sequel

184 Binding Kit 3.0 (Pacific Biosciences, Menlo Park, CA, USA). Polymerase-bound SMRTbell

185 complexes were purified with 0.6X AMPure ${ }^{\circledR}$ PB beads and sequenced on a PacBio Sequel

186 System using Sequel Sequencing Kit 3.0 and a customized setting with 4 hours of complex

187 immobilization and 20-hour movie collection. 


\section{Sequencing data and repeat sequence analysis}

191 Sequencing data repeat sequence analysis were performed following the Analysis Procedure

192 - No-Amp Data Preparation and Repeat Analysis available on the PacBio website

193 (https://www.pacb.com/wp-content/uploads/Analysis-Procedure-No-Amp-Data-

194 Preparation-and-Repeat-Analysis.pdf). Barcode demultiplexed high accuracy long reads (HiFi reads) were first generated using the PacBio SMRT Link software v8.0 or later version. HiFi reads provide accuracy of $>99.9 \%$. HiFi reads corresponding to each sample were mapped to GRCh38 human reference sequence, allowing on-target read identification and visual

198 inspection of sequencing reads using Integrative Genomics Viewer ${ }^{28}$. Repeat sequence 199 analysis was then performed using the RepeatAnalysisTools and instruction available through 200 GitHub (https://github.com/PacificBiosciences/apps-

201 scripts/tree/master/RepeatAnalysisTools) to generate various repeat sequence statistics,

\section{Amplicon long-read targeted sequencing}

204 DM1 locus was characterized in DM1 individuals 11 and 18 according to the method described 205 in Mangin et $a .{ }^{27}$ Briefly, normal and expanded CTG repeat alleles were amplified by PCR using barcoded ST300-F (5'-GAACTGTCTTCGACTCCGGG-3') and ST300-R $\quad\left(5^{\prime}-\right.$ GCACTTTGCGAACCAACGAT-3') primers. In order to enrich the SMRTbell ${ }^{\circledR}$ library with the expanded allele, the PCR products were purified using a $0.45-0.5 \mathrm{X}$ volume of AMPure ${ }^{\circledR} \mathrm{PB}$ 


\section{$\underline{\text { Small Pool PCR }}$}

215 Small pool PCR (SP-PCR) amplifications and PCR product electrophoretic analyses were 216 performed according to Gomes-Pereira et al. ${ }^{29}$. Briefly, blood DNA samples were digested 217 with HindIII enzyme and SP-PCR was performed using DM-C (5'-AACGGGGCTCGAAGGGTCCT$\left.2183^{\prime}\right)$ and DM-BR (5'-CGTGGAGGATGGAACACGGAC-3') primers ${ }^{26}$. The conditions of PCR were as 219 follows: denaturation at $96^{\circ} \mathrm{C}$ for $5 \mathrm{~min}$ followed by 30 cycles at $96^{\circ} \mathrm{C}$ for $45 \mathrm{~s}, 68^{\circ} \mathrm{C}$ for $30 \mathrm{~s}$ and 220 at $70^{\circ} \mathrm{C}$ for $3 \mathrm{~min}$ with a chase of $1 \mathrm{~min}$ at $68^{\circ} \mathrm{C}$ and $10 \mathrm{~min}$ at $72^{\circ} \mathrm{C}$. SP-PCR products were 221 loaded on a 1\% agarose gel to compare instability between DM1 patients 11 and 18 and ran at $300 \mathrm{~V}$ for $30 \mathrm{~min}$ followed by 16 hours at $160 \mathrm{~V}$. The PCR products were then transferred to GeneScreen Plus Hybridization Transfer Membrane (Perkin Elmer) and detected by hybridization using a non-radioactive method as described in Tomé et $a .^{30}$.

\section{Results}

227 Amplification-free DM1 locus enrichment in DM1 patients.

228 Amplicon-based long-read sequencing depends on PCR, which has an inherent bias towards smaller repeats. To overcome this limitation, an amplification-free long read sequencing 230 method (No-Amp) was used to specifically characterize the DM1 locus. This method utilizes 231 the CRISPR/Cas9 system to target and isolate the DNA fragment of interest from genomic DNA, 232 in combination with long-read single-molecule real-time (SMRT) sequencing. This method was 233 performed in seven DM1 patients using DNA isolated either from blood or lymphoblastoid cell 234 lines. These patients carried different CTG repeat expansion lengths that were previously 235 defined by Southern Blot and/or PCR. The number of generated on-target HiFi reads was 236 sample-dependent and ranged from 114 to 733 DMPK reads (Table 3). The CTG repeat region 237 was successfully captured in all samples, with a better result for samples \#A3, \#15969 and 
\#15841. For those samples, DNA was extracted with a high molecular weight (HMW) DNA extraction kit (New England Biolabs). Off-target reads were observed for all samples and represent an average number of approximately $54 \%$. No significant accumulation of off-target sequencing reads mapping to a specific genome location was observed in the samples

242 digested exclusively with DM1 locus guide RNAs; all the off-target sequencing reads appeared

243 to be randomly distributed across the GRCh38 reference (Figure 1). However, some specific 244 off-target reads on chromosome 2 (chr2:116779778-116785717) and chromosome 13 245 (chr13:89237016-89240938) were captured in two of the three samples digested by DM1 and 246 control locus guide RNAs (Table 2 and Figure 1). These specific off-target reads are potentially 247 produced by off-target guide RNA sequence (DMPK-crRNA2, C9orf72-crRNA1 and C9orf72248 crRNA2) matches with 2-4 mismatched bases (data not shown). Off-target as well as control 249 reads were filtered out during the bioinformatics analysis and did not affect analysis of DM1250 specific reads. For samples \#L1 and \#L3 carrying the largest CTG repeat sizes, number of highly 251 accurate long reads from the expanded allele was lower than for the other samples in this 252 study. The lower sequencing yields for these large alleles are likely the results of reduced 253 efficiency of immobilization of DNA-polymerase complex into the SMRT cell via diffusion and 254 lower raw reads to HiFi reads conversion efficiency due to significantly longer template 255 lengths.

257 Targeted amplification-free long-read sequencing improves the accuracy of CTG repeat size 258 measurement

259 This is the first time that amplification-free targeted sequencing has been described to 260 generate long-read sequencing data analysis for the DM1 locus using the RepeatAnalysisTools 261 developed by PacBio (see Materials and Methods for detail). By amplification-free sequencing, 
the number of CTG repeats was quantified in seven DM1 patients and ranged from 141 to and 495 in patients \#11 and \#18, respectively, which was a similar range to that observed for median CTG repeat size was estimated to be 563 and 927 CTG in individuals \#L1 and \#L3, respectively, by the amplicon sequencing experiment, and 1378 CTG and 1287 CTG by the No-

Amp experiment (Table 4). These results strongly suggest that amplicon sequencing is

271 subjected to the inherent preferential amplification of the smallest repeats, particularly in

272 individuals \#L1 and \#L3 carrying the largest inherited expanded alleles. Thus, PCR may

273 introduce a confounding and undesirable bias in repeat sizing in DM1 patients. The median

274 CTG repeat size for the expanded allele in samples \#A3 and \#15969, based on No-Amp

275 targeted sequencing, is consistent with previous Amplicon targeted sequencing data from

276 Mangin et $a .^{27}$. Interestingly, the number of repeats in patient \#15841 was estimated to be

277467 CNG repeats by the No-Amp method. This size estimate could not be done before by PCR

278 because it failed several times.

280 Targeted amplification-free long-read sequencing exhibits somatic mosaicism in DM1 patients

281 The distribution of CNG repeats was analyzed in individuals \#11 and \#18 carrying less than 500

282 interrupted CNG repeats by targeted long-read sequencing as well as by Small-pool PCR which

283 is usually used to estimate somatic mosaicism in DM1 patients. All these methods, including

284 SP-PCR, showed that somatic mosaicism was lower in individual \#18 than in individual \#11

285 (Figure 2). Despite similar somatic mosaicism observed with the different methods, a PCR bias 
where the shortest alleles were overrepresented using long read sequencing of PCR products

(Amp) is clearly observed. Interestingly, approximately 200 single molecule HiFi reads of the

expanded allele appear sufficient to accurately estimate somatic mosaicism in patients by no-

Amplification targeted sequencing (Figure 2). Somatic mosaicism was also observed in blood

from patients \#L1 and \#L3 carrying more than 1000 pure CTG repeats at the DM1 locus (Figure

3A). Despite a limited number of HiFi reads obtained, no-Amplification targeted sequencing

provides a better estimate of CTG repeat number distribution in patients with large expansions where PCR bias is extremely high using amplification targeted sequencing. To and \#15969 exhibit somatic mosaicism to varying degrees, with the highest being observed in patient \#A3 (Figure 3B).

299 In the literature, the interrupted expanded allele has recently been shown to be a key factor

300 in the severity of DM1 symptoms and the age of onset of these symptoms ${ }^{1,13-22}$. The

301 identification of interruptions in DM1 patients at diagnosis is therefore important to improve 302 genetic counseling in DM1. Using the no-amplification method, we analyzed repeat expansion 303 sequences to identify interrupted expanded alleles in DM1 patients. Samples \#11 and \#18 304 showed interruptions at the $3^{\prime}$ end of the CTG repeat expansion, which were also detected by 305 TP-PCR (Figure 4 and data not shown). Two CCG interruptions and four CCG interruptions were 306 identified in samples \#11 and \#18, respectively, by targeted sequencing of the DM1 locus, 307 identification which was impossible with other gold standard methods (Figure 4). 308 Interestingly, a single CAG repeat interruption at the $5^{\prime}$ end of the CTG repeat expansion was 309 also identify in the blood-derived lymphoblastoid cell line of sample \#A3 (Figure 4). In sample 
\#15841, amplification of the expanded allele failed by PCR, making it impossible to analyze

311 the CNG repeat expansion sequence. By targeted no-amplification sequencing, we overcame

312 this limitation and identified approximately 78 CCG interruptions in this patient's mutated

313 allele located both at the $3^{\prime}$ end of the CTG repeat expansion and in the middle of the sequence

314 (Figure 4).

Identification of DM1 families carrying CCG-enriched expanded allele

DM1 patients in families 1 and 2 were identified at the neuro-myology department (Myology

318 Institute Paris) as atypical DM1 patients in whom mild or no muscle symptoms were reported.

319 In addition, no cardiac and severe respiratory abnormalities were observed (Figure 5 and Table

320 5). In particular, individual \#14 is completely asymptomatic even after 12 years of follow up.

321 Individual \#14 has neither motor weakness nor myotonia, even on electromyography.

322 Respiratory and cardiac investigations remain normal. These clinical aspects differ from what

323 is usually observed in DM1 patients with similar age and CTG repeat size (ranging from 360 to

3241000 by Southern blotting) ${ }^{4}$. To better characterize the DMPK mutation in DM1 members of

325 these two families, targeted sequencing without amplification was performed in individuals

$326 \# 1, \# 14, \# 16$ and \#17 where conventional PCR failed. The amplification free DM1 locus

327 enrichment is shown in table 6 where only 8 expanded allele HiFi reads were obtained in

328 sample \#1, making it difficult to estimate repeat size and somatic mosaicism in this patient

329 (Table 6). The estimated size of the repeats in samples \#14, \#16, and \#17 was 299, 224 and

330360 CNG repeats, respectively, which is lower than what was estimated by Southern blot (360,

331430 and 1000 CNG repeats, respectively) (Table 7). These results may be explained by an

332 insufficient number of HiFi reads obtained for each sample, particularly for samples \#16 and

333 \#17. Strikingly, large expanded alleles enriched in CCG triplet repeats were identified, which 
explains the difficulties in amplifying the CNG repeat expansions in these patients (Figure 5).

hexamer (CTGCCG) appears to stabilize the repeat in the blood of patient \#14 (aged 35 years,

Figure 6). In family 2, patients \#16 and \#17 carry an expanded allele with more than $85 \%$ CCG

340 interruptions. The major allele structure observed in \#16 is

341 (CTG)7(CCG)(CTG)3(CCG)197(CTG)16 and differs from that observed in her daughter \#17,

342 where the major allele structure is (CTG)7(CCG) (CTG)3(CCG)330(CTG)19. No-Amp sequencing

343 results showed a maternal CNG repeat expansion between individuals \#16 and \#17, suggesting

344 that DM1 expanded allele with block CCG interruptions is unstable across generations in this

345 family. Furthermore, somatic mosaicism was observed in these two patients with two

346 different distributions of CTG repeat number (Figure 6). The low number of HiFi reads did not

347 allow an accurate estimate of somatic mosaicism in these patients (Table 6). However, for the

348 first time, a new variant at the DM1 locus composed of more than $85 \%$ CCG repeats was

349 identified using the No-Amp targeted sequencing method.

\section{Discussion}

352 In DM1, current routine molecular approaches do not provide simultaneous precise 353 characterization of the size and the sequence of the DM1 mutation with precision as well as

354 the dynamics of repeat instability in DM1 patients (Table1). These parameters are important 355 DM1 disease modifiers ${ }^{3}$. In some cases, incomplete genetic analyses may lead to an inaccurate 356 prognosis in DM1 patients. New genetic tests for DM1 are becoming essential to solidify the 357 diagnosis, aid prognosis and improve care with shorter time frames. Today, the diagnostic 
delays remain too long for DM1 families who are often diagnosed at the birth of their child

with a severe form of the pathology. Recently, we have shown that PacBio long-read

sequencing from amplicons can sequence large CTG repeat expansions in DM1 patients and

detect CTG repeat interruptions ${ }^{27}$. Nevertheless, this approach remains limited by PCR large or interrupted expanded alleles ${ }^{4,10}$. This number is certainly underestimated by the fact

that the size and sequence of the CTG repeat expansion is not systematically determined

367 during diagnosis. Amplification-free targeted sequencing allowed highly accurate sequence

368 determination of hundreds of expanded DNA molecules in several DM1 samples.

369 We have observed up to 50\% DM1 on-target rate in HiFi sequencing reads when targeting only

370 the DM1 locus with the Amplification-free targeted sequencing method. The on-target rate is

371 affected by the quality of genomic DNA where high molecular weight DNAs provide the

372 highest no-amp sequencing yield in this study (Table 3). Recently, it has also shown that high

373 molecular weight DNA, yielding DNA ranging from 50 to 300+ kilobases in length, generates

374 the highest quality long-read sequencing data of complex metagenomes ${ }^{31}$. The amplification-

375 free targeted sequencing method is also sensitive to the origin of DNA samples. Variable

376 results with DNA samples extracted from different tissues or cell types have been observed

377 (data not shown). In order to continue improving the methodology in the future, additional

378 studies to understand how different DNA sources and extraction methods are affecting this

379 targeted sequencing application are needed.

380 During library preparation without amplification, a distribution close to $50 \%-50 \%$ of normal 381 and mutated sequencing reads was observed in most sequencing data. However, an 
overrepresentation of the normal allele in patients with the largest expansion was observed.

This phenomenon can be explained by lower HiFi read conversion rate because of the sequencing read length limitation and lower immobilization efficiency of DNA-polymerase complex on the Sequel sequencing chip. Long DNA molecules also have higher probability to carry damages susceptible to exonuclease degradation during sequencing library preparation.

387 The combination of these factors could lead to non $50 \%-50 \%$ distribution of sequencing reads 388 on normal and expanded alleles. Although including circular consensus (CCS) reads with 389 predicted read quality below QV20 (accuracy $<99 \%$ ) in data analysis could recover additional 390 reads with long repeat expansion, the option was not explored in this study to strengthen the 391 identification of CNG interruptions. Improved long template immobilization efficiency and 392 better sequencing read length on the newer SMRT sequencing platform could help overcome 393 these limitations and provide more even coverages between normal and expanded alleles.

394 Removal of the PCR amplification step improves the accuracy of the measurement of the 395 inherited CTG repeat expansion as well as the measurement of somatic mosaicism observed 396 in blood or immortalized cells in most DM1 patients. Using No-Amplification targeted 397 sequencing, we were also able to accurately detect interruptions and determine their number 398 in blood and/or immortalized cells of all DM1 patients, regardless of the number of reads.

399 For the first time, DM1 patients with an expansion composed of more than $85 \%$ CCG 400 associated with mild or no muscle symptoms and the absence of cardiac and severe 401 respiratory abnormalities were identified by this method. This finding is consistent with recent 402 studies showing that interrupted expanded alleles are associated with a decrease of the 403 severity of DM1 symptoms ${ }^{10,11,17,19-21}$. It also raises question about systematic analysis of 404 interruptions in DM1 patients. Altogether, our results confirm the need to revisit molecular 
diagnosis with No-Amp long-read sequencing to improve genotype-phenotype correlations and thus genetic counseling and prognosis in DM1.

407 Interestingly, heterogeneity in the sequence of the expansion, characterized by different expansion lengths and varying number of repeat interruptions, was detected in families 1 and

2. The heterogeneity in the number and type of interruptions observed in expanded

410 interrupted alleles across generations (family 2) and in the sibling blood of family 1 suggests

411 instability of the interruptions despite the fact that the same interruptions can stabilize the

412 pure CTG repeat expansion ${ }^{13-18,22}$. The emergence of new interruptions in the expansion may

413 result from multiple processes including DNA polymerase errors during replication, error-

414 prone DNA repair or recombination. This method opens new avenues to understand the role

415 of interruptions in DM1. The different mechanisms involved in the formation of CNG

416 interruptions and instability of CTG repeats at the DM1 locus remain unknown and need to be

417 identified in patients carrying the interrupted expanded allele using new models.

418 In conclusion, this method allows to simultaneously obtain high resolution information on the

419 number of repeats, a complete and accurate sequence and a measure of somatic mosaicism

420 even for long repeats in the same assay. This approach is highly relevant in DM1, which is one

421 of the most complex trinucleotide disorders associated with the largest expansion and with

422 high genetic and clinical variability. No-amplification targeted sequencing gives us the

423 opportunity to better understand the dynamics of CTG repeat instability and genotype-

424 phenotype association in DM1. This method will be extremely useful to validate innovative

425 therapeutic strategies aiming at decreasing the CTG.CAG repeat length and thus stop the 426 progression of the disease in DM1 patients. 
bioRxiv preprint doi: https://doi.org/10.1101/2022.02.22.481438; this version posted February $22,2022$. The copyright holder for this

preprint (which was not certified by peer review) is the author/funder, who has granted bioRxiv a license to display the preprint in perpetuity. It is made available under aCC-BY-NC-ND 4.0 International license.

\section{Acknowledgements}

430 The authors would like to thank the DM1 patients and colleagues at the Myology Centre for

431 Research, Curie Institute and PacBio for helpful discussions and comments. We specially thank

432 Sonia Lameiras, Sylvain Baulande, Tina Alaeitabar, Lori Aro, Deborah Moine and David Stucki

433 for their active involvement in this project. This work was supported by the Institut National

434 de la Santé et de la Recherche Médicale, Sorbonne Université, Institut de Myologie

435 (Accelerator project) and the 2019 Targeted Sequencing SMRT Grant.

436

437

438

439

440

441

442

443

444

445

446 


\section{References}

448 1. Khristich AN, Mirkin SM. On the wrong DNA track: Molecular mechanisms of repeat449 mediated genome instability. Journal of Biological Chemistry, 2020, 295:4134-70

$450 \quad$ 2. Harper PS. Myotonic dystrophy. London, W.B. Saunders, 2001

451 3. Tomé S, Gourdon G. DM1 Phenotype Variability and Triplet Repeat Instability:

452 Challenges in the Development of New Therapies. IJMS, 2020, 21:457

453 4. De Antonio M, Dogan C, Hamroun D, Mati M, Zerrouki S, Eymard B, Katsahian S, Bassez

454 G. Unravelling the myotonic dystrophy type 1 clinical spectrum: A systematic registry-based 455 study with implications for disease classification. Revue Neurologique, 2016, 172:572-80

456 5. Genet AJH. Review Article: Anticipation in Myotonic Dystrophy: New Light on an Old 457 Problem Peter S. Harper, Helen G. Harley, William Reardon, and Duncan J. Shaw, n.d.:7

458 6. Kamsteeg E-J, Kress W, Catalli C, Hertz JM, Witsch-Baumgartner M, Buckley MF, van 459 Engelen BGM, Schwartz M, Scheffer H. Best practice guidelines and recommendations on the 460 molecular diagnosis of myotonic dystrophy types 1 and 2. Eur J Hum Genet, 2012, 20:1203-8

461 7. Savić Pavićević D, Miladinović J, Brkušanin $M$, Šviković S, Djurica S, Brajušković G, 462 Romac S. Molecular Genetics and Genetic Testing in Myotonic Dystrophy Type 1. BioMed 463 Research International, 2013, 2013:1-13

464 8. Morales F, Couto JM, Higham CF, Hogg G, Cuenca P, Braida C, Wilson RH, Adam B, del 465 Valle G, Brian R, Sittenfeld M, Ashizawa T, Wilcox A, Wilcox DE, Monckton DG. Somatic 466 instability of the expanded CTG triplet repeat in myotonic dystrophy type 1 is a heritable quantitative trait and modifier of disease severity. Human Molecular Genetics, 2012, 21:3558- 
expanded CTG repeat in myotonic dystrophy type 1 are associated with variation in age-atonset. Human Molecular Genetics, 2020, 29:2496-507

10. Cumming SA, Jimenez-Moreno C, Okkersen K, Wenninger S, Daidj F, Hogarth F, Littleford R, Gorman G, Bassez G, Schoser B, Lochmüller H, van Engelen BGM, Monckton DG, on behalf of the OPTIMISTIC Consortium. Genetic determinants of disease severity in the myotonic dystrophy type 1 OPTIMISTIC cohort. Neurology, 2019, 93:e995-1009

11. Overend G, Légaré C, Mathieu J, Bouchard L, Gagnon C, Monckton DG. Allele length of the DMPK CTG repeat is a predictor of progressive myotonic dystrophy type 1 phenotypes. Human Molecular Genetics, 2019, 28:2245-54

12. Peric S, Pesovic J, Savic-Pavicevic D, Rakocevic Stojanovic V, Meola G. Molecular and Clinical Implications of Variant Repeats in Myotonic Dystrophy Type 1. IJMS, 2021, 23:354

13. Braida C, Stefanatos RKA, Adam B, Mahajan N, Smeets HJM, Niel F, Goizet C, Arveiler B, Koenig M, Lagier-Tourenne C, Mandel J-L, Faber CG, de Die-Smulders CEM, Spaans F, Monckton DG. Variant CCG and GGC repeats within the CTG expansion dramatically modify mutational dynamics and likely contribute toward unusual symptoms in some myotonic dystrophy type 1 patients. Human Molecular Genetics, 2010, 19:1399-412

14. Musova Z, Mazanec R, Krepelova A, Ehler E, Vales J, Jaklova R, Prochazka T, Koukal P, Marikova T, Kraus J, Havlovicova M, Sedlacek Z. Highly unstable sequence interruptions of the CTG repeat in the myotonic dystrophy gene. Am J Med Genet, 2009, 149A:1365-74

15. Botta A, Rossi G, Marcaurelio M, Fontana L, D'Apice MR, Brancati F, Massa R, G Monckton D, Sangiuolo F, Novelli G. Identification and characterization of 5' CCG interruptions in complex DMPK expanded alleles. Eur J Hum Genet, 2017, 25:257-61

16. The Scottish Myotonic Dystrophy Consortium, Cumming SA, Hamilton MJ, Robb Y, Gregory H, McWilliam C, Cooper A, Adam B, McGhie J, Hamilton G, Herzyk P, Tschannen MR, 

of CCG, CTC and CGG interruptions within DMPK alleles in the pathogenesis and molecular diagnosis: Variant interruptions in pathogenesis and molecular diagnosis of DM1. Clin Genet, 2017, 92:355-64

18. Tomé S, Dandelot $E$, Dogan $C$, Bertrand A, Geneviève $D$, Péréon $Y$, Simon $M$, Bonnefont J-P, Bassez G, Gourdon G. Unusual association of a unique CAG interruption in 5' of DM1 CTG repeats with intergenerational contractions and low somatic mosaicism. Human Mutation, 2018, 39:970-82

19. Wenninger S, Cumming SA, Gutschmidt K, Okkersen K, Jimenez-Moreno AC, Daidj F,

508 Associations Between Variant Repeat Interruptions and Clinical Outcomes in Myotonic

510 20. Miller JN, van der Plas E, Hamilton M, Koscik TR, Gutmann L, Cumming SA, Monckton

511 DG, Nopoulos PC. Variant repeats within the DMPK CTG expansion protect function in 512 myotonic dystrophy type 1 . Neurol Genet, 2020, 6:e504

513 21. Ballester-Lopez A, Koehorst E, Almendrote M, Martínez-Piñeiro A, Lucente G, Linares514 Pardo I, Núñez-Manchón J, Guanyabens N, Cano A, Lucia A, Overend G, Cumming SA, 515 Monckton DG, Casadevall T, Isern I, Sánchez-Ojanguren J, Planas A, Rodríguez-Palmero A, 516 Monlleó-Neila L, Pintos-Morell G, Ramos-Fransi A, Coll-Cantí J, Nogales-Gadea G. A DM1 family 517 with interruptions associated with atypical symptoms and late onset but not with a milder 518 phenotype. Human Mutation, 2020, 41:420-31 

polymorphism in the MSH3 mismatch repair gene is associated with the levels of somatic instability of the expanded CTG repeat in the blood DNA of myotonic dystrophy type 1 patients. DNA Repair, 2016, 40:57-66

24. Flower M, Lomeikaite V, Ciosi M, Cumming S, Morales F, Lo K, Hensman Moss D, Jones

L, Holmans P, Monckton DG, Tabrizi SJ, TRACK-HD Investigators, Kraus P, Hoffman R, Tobin A,

528 Borowsky B, Keenan S, Whitlock KB, Queller S, Campbell C, Wang C, Langbehn D, Axelson E, 529 Johnson H, Acharya T, Cash DM, Frost C, Jones R, Jurgens C, 't Hart EP, van der Grond J, Witjes530 Ane M-NN, Roos RAC, Dumas EM, van den Bogaard SJA, et al. MSH3 modifies somatic 531 instability and disease severity in Huntington's and myotonic dystrophy type 1. Brain, 2019, $532 \quad 142: 1876-86$

533 25. Warner JP, Barron LH, Goudie D, Kelly K, Dow D, Fitzpatrick DR, Brock DJ. A general 534 method for the detection of large CAG repeat expansions by fluorescent PCR. Journal of 535 Medical Genetics, 1996, 33:1022-6

536 26. Monckton DG, Wong L-JC, Ashizawa T, Caskey CT. Somatic mosaicism, germline 537 expansions, germline reversions and intergenerational reductions in myotonic dystrophy 538 males: small pool PCR analyses. Human Molecular Genetics, 1995, 4:1-8

539 27. Mangin A, de Pontual L, Tsai Y-C, Monteil L, Nizon M, Boisseau P, Mercier S, Ziegle J, 540 Harting J, Heiner C, Gourdon G, Tomé S. Robust Detection of Somatic Mosaicism and Repeat 541 Interruptions by Long-Read Targeted Sequencing in Myotonic Dystrophy Type 1. IJMS, 2021, 
28. Robinson JT, Thorvaldsdóttir H, Winckler W, Guttman M, Lander ES, Getz G, Mesirov

544 JP. Integrative genomics viewer. Nat Biotechnol, 2011, 29:24-6

545 29. Gomes-Pereira M, Bidichandani SI, Monckton DG. Analysis of Unstable Triplet Repeats

546 Using Small-Pool Polymerase Chain Reaction. Trinucleotide Repeat Protocols, vol. 277, New

547 Jersey, Humana Press, 2004, pp. 061-76

548 30. Tomé S, Nicole A, Gomes-Pereira M, Gourdon G. Non-Radioactive Detection of

549 Trinucleotide Repeat Size Variability. PLoS Curr, 2014

550 https://doi.org/10.1371/currents.md.ad50113b899fa1352ce70c087eead706

551 31. Florian Trigodet, Lolans Karen, Fogarty Emily, Shaiber Alon, Morrison Hilary, Barreiro

552 Luis, Jabri bana, Eren A Murat. High molecular weight DNA extraction strategies for long-read

553 sequencing of complex metagenomes. Molecular Ecology Resources, n.d.

554 
Table 1: Genetic testing strategy for DM1

\begin{tabular}{|c|c|c|c|}
\hline Information & PCR & Southern blot & TP-PCR \\
\hline Mutation Status & $\sqrt{ }$ & $\sqrt{ }$ & $\sqrt{ }$ \\
\hline $\begin{array}{c}\text { CTG repeat size } \\
\quad<200-300\end{array}$ & $\sqrt{ }$ & $\sqrt{ }$ & $\sqrt{ }$ \\
\hline $\begin{array}{c}\text { Large CTG repeat } \\
\text { size }\end{array}$ & $\mathrm{x}$ & $\sqrt{ }$ & $\mathrm{x}$ \\
\hline Structural variants & $\mathrm{x}$ & $\mathrm{x}$ & $\mathrm{x}$ \\
\hline Somatic mosaicism & $\mathrm{x}$ & $\mathrm{x}$ & $\mathrm{x}$ \\
\hline
\end{tabular}


Table 2: crRNA and genomic coordinate for DMPK and control loci (* Fragment size after CRISPR/Cas9 digestion)

\begin{tabular}{|c|c|c|c|c|}
\hline Locus & crRNA1 & crRNA2 & GRCh38 & $\begin{array}{l}\text { Size of the fragment* (bp) } \\
\text { (Non-expanded allele) }\end{array}$ \\
\hline \multirow{2}{*}{$D M P K$} & \multirow{2}{*}{ CCCCATCGGGACAACGCAGA } & \multirow{2}{*}{ GGGCGTGTATAGACACCTGG } & Chromosome 19 & \multirow{2}{*}{2328} \\
\hline & & & (45769144-45771505) & \\
\hline$H T T$ & CTTATTAACAGCAGAGAACT & TAAACTTTGAAGACGAGACA & (3072601-3076440) & 3806 \\
\hline C9orf72 & TTGGTATTTAGAAAGGTGGT & GGAAGAAAGAATTGCAATTA & Chromosome 9 & 3639 \\
\hline$F M R 1$ & CGCGCGTCTGTCTTTCGACC & CCTTTATGCAAAGTTAGCTC & (147911739-147915434) & 3662 \\
\hline
\end{tabular}

586 
595 Table 3: HiFi reads in DM1 patients (**Includes DMPK and control loci reads)

\begin{tabular}{|c|c|c|c|c|c|c|c|}
\hline Individuals & \#11 & \#18 & \#L1 & \#L3 & \#A3 & $\# 15969$ & $\# 15841$ \\
\hline Tissue & Blood & Blood & Blood & Blood & Lymphoblastoid cells & Lymphoblastoid cells & Lymphoblastoid cells \\
\hline DNA extraction & $\mathrm{N} / \mathrm{D}$ & N/D & $\mathrm{N} / \mathrm{D}$ & $\mathrm{N} / \mathrm{D}$ & $\begin{array}{l}\text { Monarch Genomic } \\
\text { DNA Purification Kit } \\
\text { (NEB) }\end{array}$ & $\begin{array}{c}\text { Monarch Genomic DNA } \\
\text { Purification Kit (NEB) }\end{array}$ & $\begin{array}{l}\text { Monarch Genomic DNA } \\
\text { Purification Kit (NEB) }\end{array}$ \\
\hline HiFi reads & 841 & 1041 & 836 & 3731 & 7405 & 11201 & 4429 \\
\hline DMPK locus & 452 & 352 & 332 & 114 & 569 & 733 & 597 \\
\hline Control loci & none & none & none & none & 4522 & 3073 & 3218 \\
\hline Expanded allele & 206 & 205 & 65 & 21 & 303 & 398 & 361 \\
\hline Non-Expanded allele & 246 & 147 & 262 & 93 & 266 & 335 & 236 \\
\hline$\%$ reads on-target & 53,7 & 33,8 & 39,71 & 3,06 & $68,75^{* *}$ & $33,98 * *$ & $86,14^{* *}$ \\
\hline$\%$ reads off-target & 46,3 & 66,2 & 60,29 & 96,94 & 31,25 & 66,02 & 13,86 \\
\hline
\end{tabular}

596

597 
bioRxiv preprint doi: https://doi.org/10.1101/2022.02.22.481438; this version posted February 22, 2022. The copyright holder for this

preprint (which was not certified by peer review) is the author/funder, who has granted bioRxiv a license to display the preprint in perpetuity. It is made available under aCC-BY-NC-ND 4.0 International license. 
Table 4: CTG repeat size and interruptions in DM1 patients

\begin{tabular}{|c|c|c|c|c|c|c|c|}
\hline Individuals & $\# 11$ & $\# 18$ & \#L1 & \#L3 & \#A3 & $\# 15969$ & $\# 15841$ \\
\hline Tissue & Blood & Blood & Blood & Blood & Lymphoblastoid cells & Lymphoblastoid cells & Lymphoblastoid cells \\
\hline $\begin{array}{c}\text { Age at sample } \\
\text { (Years) }\end{array}$ & 20 & 42 & 29 & 26 & 40 & 36 & 58 \\
\hline $\begin{array}{c}\text { Median Expanded } \\
\text { allele } \\
\text { (No-Amp) } \\
\end{array}$ & 516 & 495 & 1378 & 1287 & 141 & 158 & 467 \\
\hline $\begin{array}{c}\text { Median Expanded } \\
\text { allele } \\
\text { (Amplicons) } \\
\end{array}$ & 468 & 460 & 563 & 927 & $\# \mathbf{N} / \mathbf{A}$ & $\# \mathbf{N} / \mathbf{A}$ & Failed \\
\hline $\begin{array}{l}\text { Interruptions } \\
\text { (No-Amp) }\end{array}$ & $2 C C G$ & 4CCG & Pure & Pure & 1CAG & Pure & $78 C C G$ \\
\hline $\begin{array}{l}\text { Interruptions } \\
\text { (Amplicons) }\end{array}$ & $2 C C G$ & 4CCG & Pure & Pure & $\# \mathbf{N} / \mathbf{A}$ & $\# \mathbf{N} / \mathbf{A}$ & Failed \\
\hline $\begin{array}{l}\text { CNG repeat size } \\
\text { (Diag) }\end{array}$ & $\cong 700$ & $\cong 400$ & $>1000$ & $>1000$ & 130 & 150 & 370 \\
\hline
\end{tabular}

601

602 
bioRxiv preprint doi: https://doi.org/10.1101/2022.02.22.481438; this version posted February 22, 2022. The copyright holder for this

preprint (which was not certified by peer review) is the author/funder, who has granted bioRxiv a license to display the preprint in perpetuity. It is made available under aCC-BY-NC-ND 4.0 International license.

604 
Table 5: Clinical features in DM1 families 1 and 2

\begin{tabular}{|c|c|c|c|c|c|c|c|}
\hline & $\begin{array}{l}\text { Age sample } \\
\text { (Years) }\end{array}$ & $\begin{array}{l}\text { Age at onset } \\
\text { (Years) }\end{array}$ & $\begin{array}{c}\text { CTG repeat } \\
\text { number } \\
\text { (Southern Blot) } \\
\end{array}$ & Weakness & Myotonia & $\begin{array}{c}\text { Cardiac } \\
\text { defect }\end{array}$ & $\begin{array}{c}\text { Respiratory } \\
\text { defect }\end{array}$ \\
\hline$\# 1$ & 45 & 36 & $\mathrm{~N} / \mathrm{A}$ & $\begin{array}{l}\text { Axial, proximal, distal and } \\
\text { facial }\end{array}$ & Yes (36y) & No & $\begin{array}{c}\text { Vital capacity } \\
91 \% \text { and } \\
\text { Hypercapnia }\end{array}$ \\
\hline$\# 14$ & 35 & Asymptomatic & 360 & No & No & No & No \\
\hline$\# 16$ & 48 & 38 & 430 & Very mild facial & No & No & No \\
\hline$\# 17$ & 21 & 20 & 1000 & $\begin{array}{c}\text { Distal weakness of the } \\
\text { hand } \\
\text { (Hirayama disease) }\end{array}$ & $\sim 15 y$ & No & No \\
\hline
\end{tabular}


612 Table 6: HiFi reads in DM1 patients of families 1 and 2

613

\begin{tabular}{|c|c|c|c|c|}
\hline Individuals & $\mathbf{\# 1}$ & $\mathbf{\# 1 4}$ & $\mathbf{\# 1 6}$ & $\mathbf{\# 1 7}$ \\
\hline Hifi reads & 926 & 652 & 510 & 1916 \\
\hline DM1 locus & 158 & 192 & 127 & 471 \\
\hline Control loci & 393 & 230 & 220 & 1275 \\
\hline Expanded allele & 8 & 79 & 42 & 38 \\
\hline Non-Expanded allele & 150 & 113 & 85 & 433 \\
\hline \% reads on-target & 59,61 & 64,88 & 64,12 & 91,13 \\
\hline \% reads off-target & 40,39 & 35,12 & 35,88 & 8,87 \\
\hline
\end{tabular}

614

615

Table 7: CTG repeat size and interruptions in DM1 patients of families 1 and 2

617

\begin{tabular}{|c|c|c|c|c|}
\hline Individuals & $\# 1$ & $\# 14$ & $\# 16$ & $\# 17$ \\
\hline $\begin{array}{c}\text { Median Expanded allele } \\
\text { (CNG) }\end{array}$ & N/A & 299 & 224 & 360 \\
\hline $\begin{array}{c}\text { Interruptions } \\
\text { \% CCG repeats }\end{array}$ & $\begin{array}{c}52 \\
\text { CCG }\end{array}$ & 115 CCG & 198 CCG & 331 CCG \\
\hline CNG repeat size (Diag) & N/A & $39 \%$ & $88 \%$ & $92 \%$ \\
\hline
\end{tabular}

618

619

620

621

622

623

624

625

626

627

628 
630

\#18

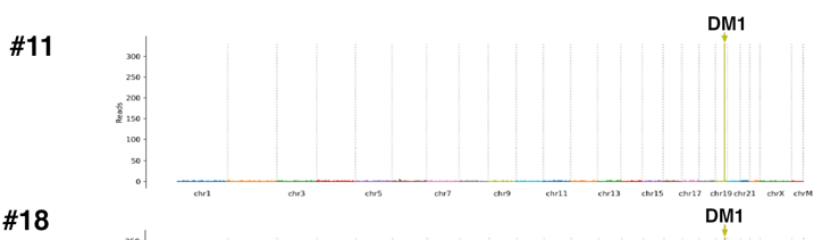

632

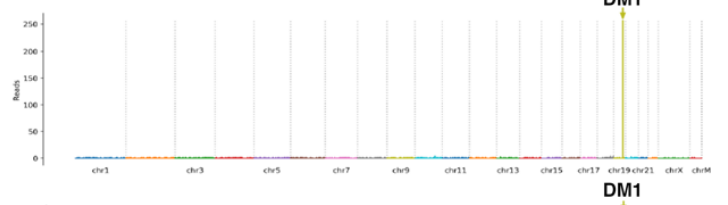

633

\#L1

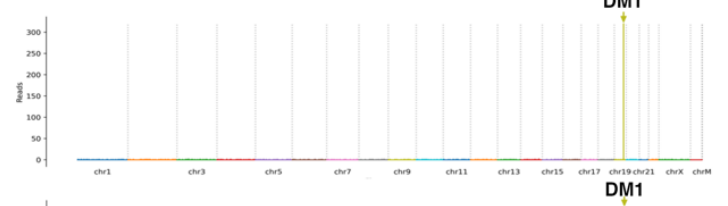

634

\#L3

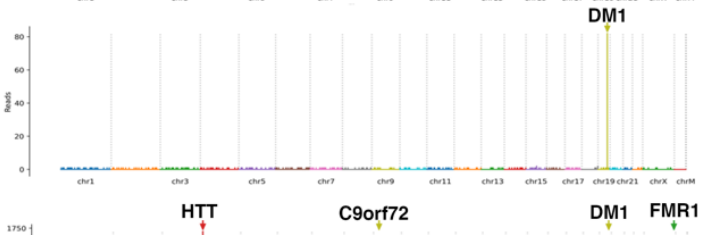

635

\#A3

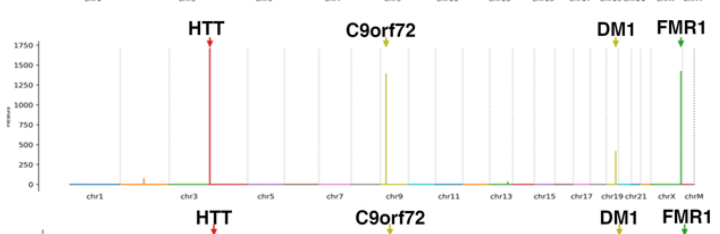

636

$\# 15969$

637

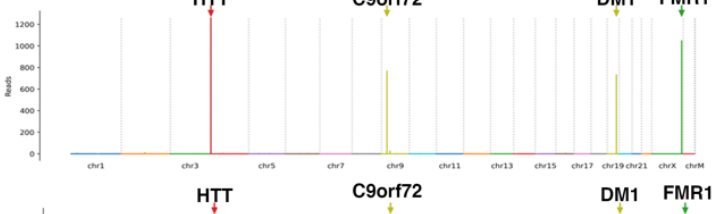

638

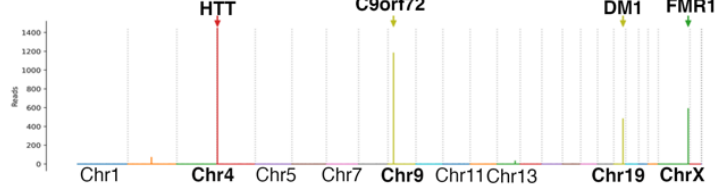

639

640 Figure 1: Genome wide coverage plots. Genome wide coverage plots for DM1 patients are

641 shown in the figure. The $y$-axis shows the number of reads and the $x$-axis spans over all the chromosomes in the Hg38 genome.

643

644 


\section{CNG repeat distribution}

A

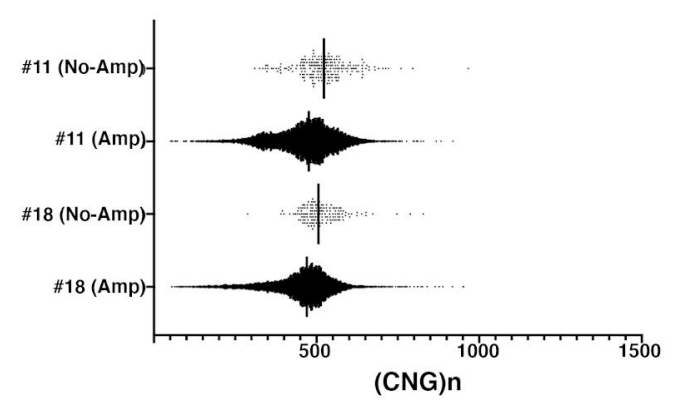

B

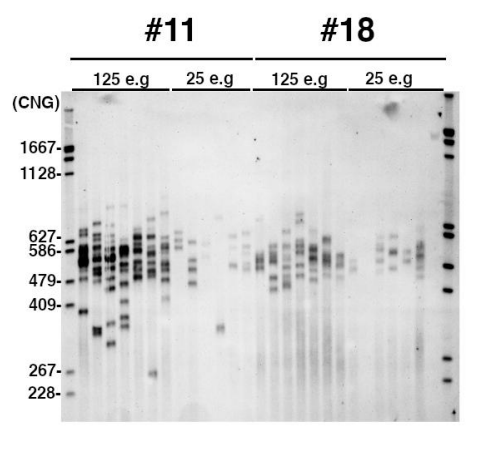

653

654 Figure 2: Somatic mosaicism in \#11 and \#18 individuals. A- The distribution of the size of the

655 repeat of each HiFi read generated by PacBio algorithm. The x-axis shows the CTG repeat 656 number. Each dot represents the CTG repeat size of one HiFi read. The black bar represents 657 the median. 10000-30000 single molecule HiFi reads of expanded allele are analyzed in the 658 Amplicon experiment whereas more than 200 single molecule HiFi reads of expanded allele 659 are analyzed in the No-Amp experiment. B- Trinucleotide repeat instability estimation by 660 Small-Pool PCR in blood samples of DM1 patients. The sizes (converted in CTG repeats) of DNA 661 molecular weight marker III, DIG-labeled are indicated on the left of the figure. About 25-125 DNA molecules are amplified for each individual. e.g means equivalent genome. 


\section{CNG repeat distribution}

A

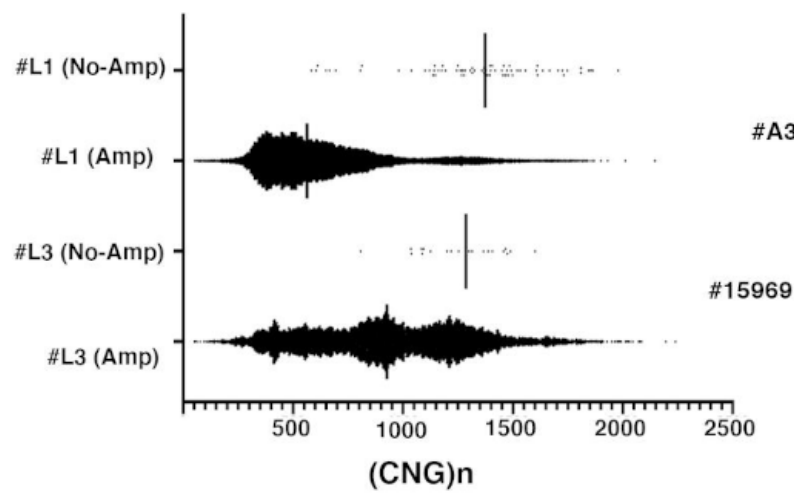

B

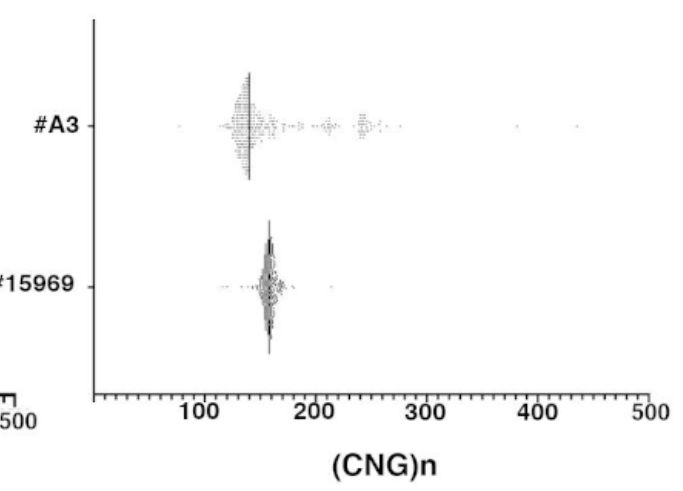

663

664 Figure 3: Distribution of the size of the repeat of each HiFi read generated by PacBio

algorithm. A- \#L1 and \#L3 individuals. B- \#A3 and \#15969 individuals. The $\mathrm{x}$-axis shows the CTG repeat number. Each dot represents the CTG repeat size of one HiFi read. The black line represents the median. 10000-30000 single molecule HiFi reads of expanded allele are analyzed in the Amplicon experiment whereas 21-398 single molecule HiFi reads of expanded allele are analyzed in the No-Amp experiment.

670

671

672

673

674

675

676 

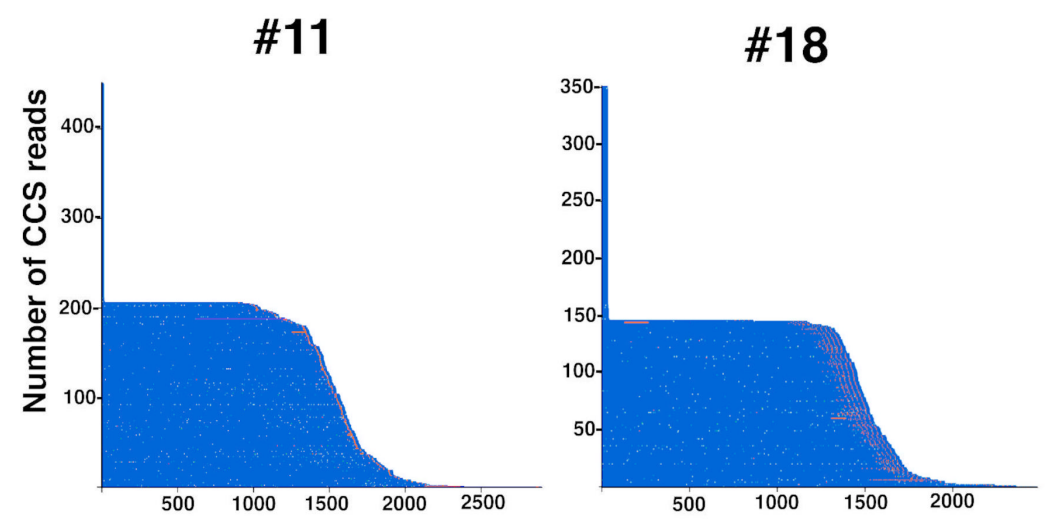

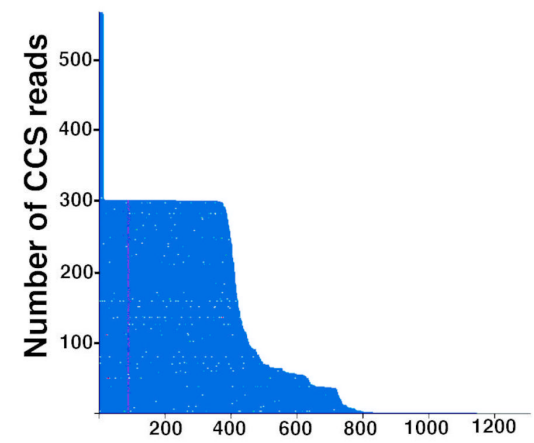

(bp)

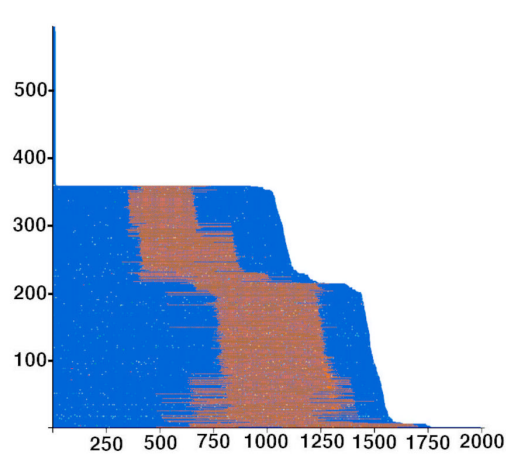

(bp)

Figure 4: Waterfall plots outline the repeat structure of the normal and expanded alleles.

697 pair (bp). 
704

705

706

707

708

709

710

711

712

713

714

715

716

717

718

719

720

721

722

723

724

725

726

\section{Family 1}

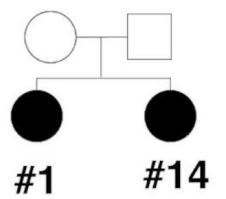

\section{Family 2}

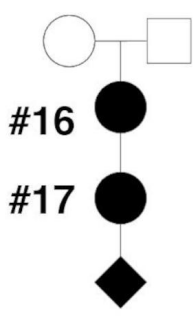

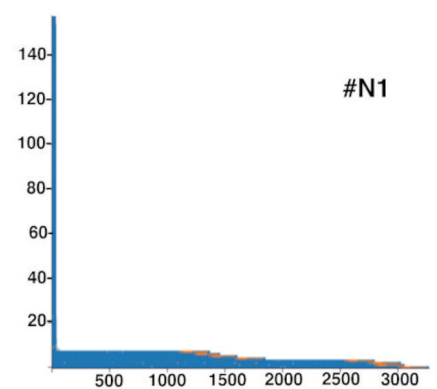
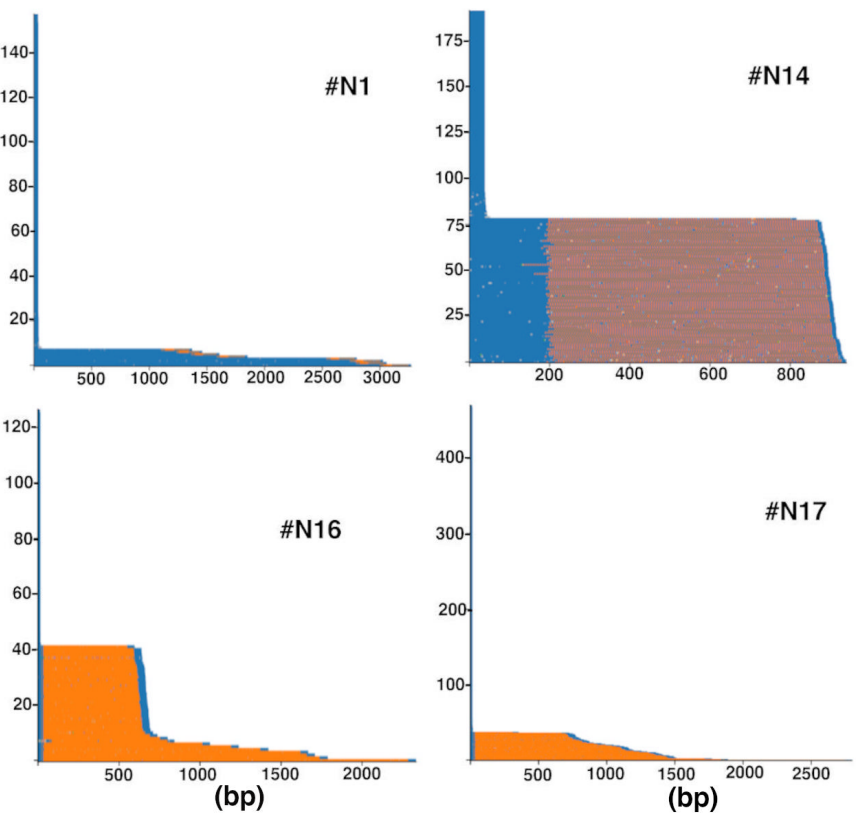

Figure 5: Waterfall plots outline the repeat structure of the normal and expanded alleles in

DM1 families 1 and 2 (PCR failed). Triplet repeat sequences are sorted from shortest to longest and each individual molecule is represented by a series of colored dots on a horizontal line. Each dot represents a single repeat unit. The CTG repeat is represented in blue whereas the CCG repeat is represented in orange. The highest peaks represent the normal allele. The $x$-axis shows the size of the repeat in base pair (bp).

.




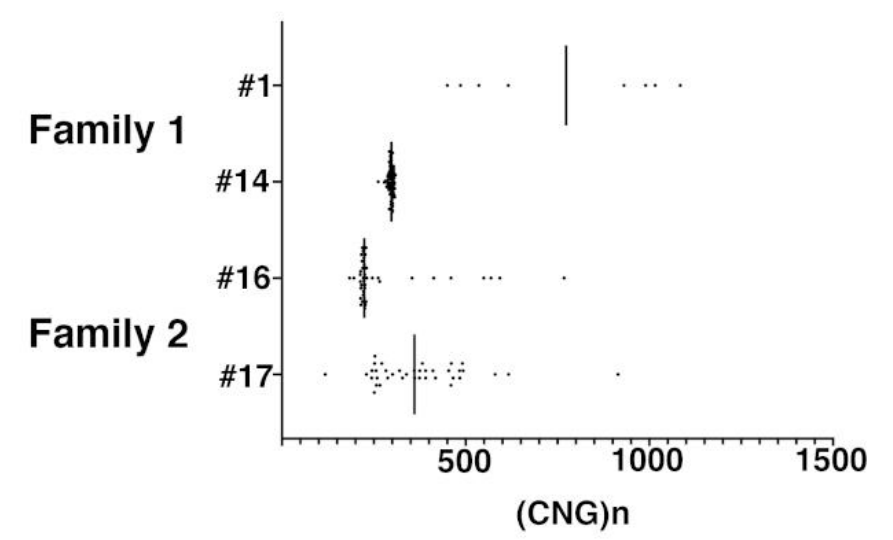

737 Figure 6: Distribution of the size of the repeat in families 1 and 2. The $x$-axis shows the CTG

738 repeat number. Each dot represents the CTG repeat size of one HiFi read. The black line

739 represents the median. 8-79 single molecule HiFi reads of expanded allele were analyzed in

740 the No-Amp experiment. 